\title{
Optimal Size \& Location of Distributed Generation using Bird Swarm Optimization with Cuckoo Search Sorting Algorithm
}

\author{
K. Sri Kumar
}

\begin{abstract}
In this paper a natural habitat inspired metaheuristic Bird Swarm Optimization algorithm is implemented with improvisations made for the development of solution for the optimal allocations and optimal size prediction problem of Dispersed generation/ Distributed Generator in a radial power system distribution system in consideration of the drawbacks in the previous algorithms both in the context of convergence time and the optimal sizing with respect to the cost analysis for operation of the system with different number of DG's installed in such a way that the optimal locations and sizes of DG's installed is finalised with highest priority to the economical operation along with the immediate priority given to the network losses along with voltage deviations. To avoid the draw backs in previous optimisation algorithm regarding accuracy and run time. Along with the Cognitive component Weighted factor Bird Swarm optimisation (CWFBSO) algorithm a new concept is introduced called DG Size tuner Such that cost effective economical installation is possible as by the size tuner it is possible to compare the losses and voltage profile within the mean difference of the optimal sizes of final allocation determined by the main algorithm i.e., CWFBSO. Obtained results using CWFBSO in determining optimal locations and sizes of DG's is capable showing good performance with less run time and convergence time and by using size tuner the optimal size selected economically with respect to less voltage deviation and minimal losses.
\end{abstract}

Keywords : Load flow, forward-backward sweep method, loss factors analysis, Voltage sensitivity factors, Cognitive component, Weighted factor, Bird Swarm optimisation, Distributed generation, Optimal location, Optimal size, Real Power losses, Size Tuner.

\section{INTRODUCTION}

One of the main reasons of emergence of DG is, Electrical energy is been lost continuously due to the presence of the resistance that exists in power distribution network, in which Distribution network losses are more in comparison with the transmission network losses [1] As distribution networks are known for the high $\mathrm{X} / \mathrm{R}$ ratio in comparison with transmission networks and also there is chance of voltage drops which results in losses along the length of the distribution network feeders. The resultant losses in power distribution system networks is been one of the most important targets to many of the utility systems all around the world.

Such that Distributed Generation (DG) technology has become one of the important aspects in power system in particular in distribution systems[2].The generator

Revised Manuscript Received on September 22, 2019

* Correspondence Author

K. SriKumar, Dept. of EEE, University College of Engineering, Kakinada, India technologies that's been classified in DG are the renewable, non renewable, fossil-fuel based power energy sources. The renewable sources of energy comprises of Photo-voltaics, Wind-turbines, Bio-fuel based, Geothermal based sources e.t.c and the fuelled DG sources are Combustion turbines and engines, fuel cells. With respect to the above all the ecological, technical and climatic beneficial factors have played a major role in Distributed Generation development $[3,4]$. According to the agreement of Kyoto on climatic changes and their impacts there are many efforts that are taken to reduce harm full gaseous emissions such as carbon, methane etc as a result the penetration of Generators[5] in distribution system network also increased and found best solution for all the problems of system losses with respect to the environmental benefits.

As in-appropriate DG locations affects the system in such a way that it increases the initial capital costs and also the Latter operation costs as a result the network losses also but if placed in appropriate positions there its the most beneficial one that it helps in keeping the voltage profiles within the limits therefore it reduces the real power flow with respect to system losses thereby improving quality of power supply to load centres along with reliable power to consumers. Hence there arises a need for a power full Optimisation algorithm techniques to provide the best suited locations and best economical sizes of allocated or installed different number of DG's to optimise the operation of the distribution power system and taking into this consideration the constraints for the operation of DG and its initial investments.

This provoked the emergence of a lot of metaheuristic techniques and optimisation algorithms that have been proposed for the ultimate optimal allocation of DG and its installed sizes. Singh[6] has introduced the Algorithm of genetics i.e., Genetic Algorithm(GA) to find locations of different number of DG's as per requirement and their sizes of individual units for the reduction of system unwanted losses and regulate power flow from the grid/substation, considering the boundary limits of voltage at each and every individual bus of the power system distribution. Moradi[7] Proposed three different methodologies for the location and size of different number of DG's that, initially he proposed GA, followed by PSO in individual and the third, a combined GAPSO method was introduced in which the most critical DG siting locations were searched and the respective sizes of DG that to be installed were optimised later by PSO. Then he compared his three methodologies are compared to each with each of the other in the form of a multiobjective function[7]. Injeti[8] proposed two methodologies of optimisation, at first calculation of the Loss

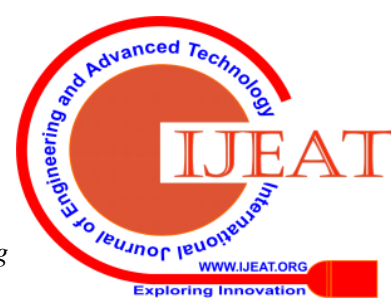


Sensitive Factors analysis(LSF) is followed for the determination of most critical buses for optimal locations thereby reducing space of search of the initially random generated locations and he followed Simulated Annealing algorithm(SA) for determining size of installing DG. Imran.A [9] introduced again the Loss factor Analysis for finding critical locations for DG units installation then Bacterial foraging methodology was applied for determining Optimal size. Likewise many researchers working on the concept of DG put concentration on different aspects for the reduction of the system real losses without taking the actual costs i.e., costs for power losses from the power drawn from substation/grid and for installation of different number of individual DG units including their maintenance. Although Some researchers [10], [11], [12] and [13] considered the mentioned costs for losses subjected to the power drawn from substation/grid, they have just worked on the improving the distribution system voltage profile thats been taken with respect to each and every bus in the system other than that in reduction of system losses simultaneously with system total operating costs.

This paper proposes a multistage Optimisation algorithm taking the drawbacks of previous techniques. The algorithm starts with load flow analysis using an easy, fast and flexible method called Forward-backward sweeping method followed by Loss factor analysis [8] for calculation of most critical locations and then using CWFPSO initially by giving maximum and minimum limits of sizes for DG there randomly generates the sizes, but there to eliminate the problem of occupancy of the search space such that Modified Cuckoo search algorithm for enhancing the Convergence rate and quality of solution as a result the search space is reduced drastically and then CWFBSO is implemented on the results from the previous stage hence more Run time is saved and in the final stage the DG Size tuner is used for best Optimal values that come from the comparison of objective values within best optimal values such that losses are reduced in consideration with the drastic cost reduction with medium Voltage regulation.

The proposed system and algorithm have been applied on standard bus systems given by IEEE i.e, 33bus, and 69bus radial distribution bus systems in MATLAB r2013 programming language software and the results found capable with good performance.

\section{MULTI-STAGE PROBLEM FORMULATION}

\section{A. MULTIPLE-OBJECTIVE FUNCTION:}

The Multiple-stage objective function in the proposed method is minimising the objective i.e., minimising the cost for the total operation which includes cost for operation of DG and its maintenance with cost for the loss of real power taken from substation for single or multi DG installations in

$$
\begin{gathered}
\text { Minimize obj }=\min \left(A_{1} D G_{I}+A_{2} V_{d}+A_{3} T o c\right) \\
\text { Where, } A_{1}, A_{2}, A_{a}-\text { Constants between }[0,1] \\
D G_{1}-\text { Real Power Losses with } D G \\
V_{d}-\text { Voltage deviation } \\
T_{0 e}-\text { Total perating cost of } D G
\end{gathered}
$$

The above objective function is evaluated for cost effective economical allocation and appropriate sizing for DG radial distribution system.

installation. The following are the basic assumptions for proceeding for the allocation and size of DG.

\section{ASSUMPTIONS:}

a. The Source bus is not considered for the placement of DG.

b. Voltage at source node is taken as 1PU.

c. At each individual bus only one DG is placed.

d. Here the maximum DG size is $75 \%$ of total apparent load demand. Whereas minimum DG size is $5 \%$ of total apparent load of system.

\section{B. POWER FLOW SOLUTION:}

The efficient algorithm for load flow solution which uses topological structure of the distribution system is implemented here. Here in considers two simple matrices, that represents the relation between bus to branch currents at each and every individual branches/lines of system and the individual branch currents at each branch to the individual bus voltages at each and every buses/nodes of the system which is known as forward-backward sweep algorithm. It has the features like robustness and it converges in almost every circumstance for distribution system, also has great potential for using it with distribution automation.

The load flow calculation starts taking the initial values of bus voltages as $1 \mathrm{Pu}$ at each bus such while in the procedure of backward sweep it attains relation between these bus voltages and injected bus currents and after convergence it starts forward sweep procedure in which it gets the actual bus voltage magnitudes at each bus of the network.

\section{DEVIATION IN VOLTAGE TRACE:}

The deviation in voltage trace is determined by the difference to the source node voltage and node voltage at each bus in the system such that the voltage deviation should be preferred least.

$$
V_{d}=V_{1}-V_{b}
$$

Where,

$$
\begin{aligned}
& V_{d}=\text { Voltage deviation } \\
& V_{1}=\text { Source bus Voltage } \\
& V_{b}=\text { Individual bus voltage }
\end{aligned}
$$

\section{TOTAL OPERATION COST:}

The total cost invested upon the operation of the generator is one of the main objective in the multi stage objective function and also it is the most advantageous point in the installations of DG. The total cost includes the cost for the maintenance of DG, DG operating costs as well as the costs for the losses when DG is installed for the Power drawn from the Sub-station. The total cost of operation should be as less as possible with respect to the losses and the voltage deviation.

$$
T_{\text {oc }}=\left(R_{1} * D G_{\text {loss }}\right)+\left(R_{2} * D G_{R}\right)
$$

Where, 


$$
\begin{aligned}
T_{00} & =\text { Total Operation cost of } D G \\
R_{1} & =\text { Rateof power drawn from Substation } \\
R_{2} & =\text { Rate of power drawn from } D G \\
D G_{\text {loss }} & =\text { Real power loss with } D G \\
D G_{R} & =\text { Active Power drawn from } D G
\end{aligned}
$$

The cost of the real power drawn from the Sub-station is considered here is $280 \mathrm{Rs}$ and the cost for the Power drawn from the DG is 350 Rs.

\section{E. TOTAL REAL POWER LOSSES:}

Here for the calculation of Real Power losses general Power loss formulae is considered,

$$
P L=\sum_{N=1}^{n} I_{N}^{2} * R_{N}
$$

Where,

$$
\begin{aligned}
P L & =\text { Total Powel Losses the system } \\
N & =\text { Individual Branch number } \\
n & =\text { Total branches in the system } \\
I_{N} & =\text { Current at each individual branch } \\
R_{N} & =\text { Resistance of each individual branch }
\end{aligned}
$$

\section{F. LOSS SENSITIVITY FACTOR ANALYSIS:}

The loss sensitivity factors [14] are essential for the selection of the most critical buses that are prone to losses such that it adds advantage for reducing the time for the search of the locating DG's at all the individual branches.

Whereas by finding the loss sensitivity factors the most critical buses are selected without the involvement of any algorithm but by means of losses. In such a way the losses at each bus that are found are placed in ascending order and top most buses of the system are given highest pripority.

$$
L S F_{n}=\left(2 * E l d_{n+1} * R_{N}\right) / V_{n+1}
$$

Where,

$$
\begin{aligned}
L S F_{n} & =\text { Loss Sensitive Factors at each branch } \\
\text { Eld }_{n+1} & =\text { Effective load beyond each bus } \\
R_{N} & =\text { Resistance at each branch } \\
V_{n+1} & =\text { Voltage at receiving end bus }
\end{aligned}
$$

\section{BIRD SWARM OPTIMISATION}

Bird Swarm Optimisation is the optimisation technique which is inspired from the nature and also called as Particle Swarm Optimisation [15]. Its one of the most efficient met-heuristic techniques that are first proposed by JamesKennedy and RusellEberhart in 1942. It is actually the bird food search phenomena which is termed as Bird flocking. In this technique a group of particles like the birds group is considered, like the bird groups finds food and their search procedure with coordination and communication is applied here for the search of optimal size of different number of DG's individually in a given distribution system.

\section{ALGORITHM}

Step 1: Initialize a random swarm population array of bird swarms with randomly generated locations and their corresponding movement with particular velocities on ' $d$ ' dimensions in the space of search of location and sizes.

Step 2: For each swarm/particle, define a fitness function in 'd' variables.

Step 3: Compare swarm/particle's fitness defined function with its individual swarm/particle's best solution also called as pbest. If present value is best than the previous solution pbest, then set presentbest value equal to the current value, and the presentbest location equal to the current location in the seaching space.

Step 4: Now Compare the defined fitness function with the swarm's overall previous best solutions. If present value is best than gbest solution that is globally best solution in comparison with all the updated present best solutions, then reset gbest to the present swarm/particle's values.

Step 5: Update the location of DG and respective velocity of each swarm/particle with respect to the globally best location.

Step 6: Repeat the Steps $3 \& 4$ till the optimum solution of locations or size gained.

Step 7: The updated globally best solution at the end of the end iteration gives the ultimate value of optimisation for location and size of different number DG's.

Step 8: Again calculation of the load flow with ultimate values of optimisation with losses reduced.

\section{MODIFIED CUCKOO BIRD SEARCH ALGORITHM}

Generally the Bird Swarm Optimisation algorithm works based upon the search for Optimal size in given $\mathrm{N}$ dimensional space such that at the initial random selection of the sizes there might be a chance of poor selection which is of no use in further procedure of finding the Optimal size of different number of DG's in selected critical location but waste of time. So to avoid such wastage of time at the initial selection of the generated random DG sizes Modified Cuckoo search algorithm[16] is used.

The Modified Cuckoo Search algorithm[16] is the behaviour of the Cuckoo bird in hatching the eggs such that it shows a different behaviour like sorting out the healthy eggs from the rotten ones to save the healthy eggs from rotting. Likewise here the algorithm is applied to sort out the best pairs of defined fitness optimisation values and the worst pairs of fitness values to objective function.

$$
\left[\begin{array}{cc}
O b j 1 & F 1 \\
O b j 2 & F 2 \\
\vdots & \vdots \\
O b j I & F I
\end{array}\right]
$$

Where,

$$
\text { Fitness value } 1(\mathrm{~F} 1)=\frac{1}{1+o b_{j}}
$$

$\mathrm{F} 1, \mathrm{~F} 2, \ldots \ldots, \mathrm{FI}$ are the fitness values that calculated from objective function taking each pair of randomly generated DG sizes where FI is the Fitness values of last iteration. And $\mathrm{Obj} \mathrm{I}$ is the objective function calculated from the last pair of last iteration. In such a way the best pairs and worst pairs are sorted by comparing the fitness values of each immediate pair and the value which greater than the other will be kept and the other values is 
discarded. As a result the initial pairs of best values will be given to the main algorithm for further procedure of finding the Optimal values of DG size.

As a result there is a lot of saving the run time by the elimination of worst pairs of randomly generated sizes at the initial step of the PSO algorithm.

\section{SIZE MODULATOR}

The drawback in all the metaheuristic techniques like Bird Swarm Optimisation is the final accuracy of the Ultimate Optimum values DG size And location which depends on initial generation if random values even though the Modified Cuckoo Bird search methods helps in reducing the search space, still there the final values depends on the random pairs left after sorting. So even though search space is reduced and time is saved for iterations still there is a need to increase the accuracy, for which there is a need for method to increase the accuracy for which in this paper a DG Size modulator is introduced which gives scope for the comparison of different values nearer in value with the final optimum size such that accurate size can be taken and also there is chance of selecting most economical DG size in cost perspective related to losses, DG generation size with consideration of voltage deviation.

\section{ALGORITHM:}

Step 1: Take the final resultant Optimum location and size from the BSO.

Step 2: Input position of DG as the final Optimal location from BSO.

Step 3: DG size is varied from minimal/least defined value to the maximum/highest defined value (Optimum size from BSO) in such a way that

DGmin $=$ Final Optimum size from BSO - A \&

DGmax $=$ Final Optimum size from $\mathrm{BSO}+\mathrm{B}$

Where, A \& B are values dependent on number of DG's and the type of system.

Step 4: Iterations start and for every iteration DG size is varied from DGmin to DG max in step of difference 1 when size converted from per unit value to base value, and the respective size is given input to the Objective function with the input position taken from Optimum location to BFS.

Step 5: Now the voltage magnitudes and power losses are calculated with given input and is compared to the Optimum values from BSO for minimum or equal values of objective function to the optimum loss from BSO.

Step 6: The modulation goes on until the Optimum size becomes larger the given input size or when it reaches maximum iteration (A + B iterations).

Step 7: Thus these final Optimum Position, Optimum size and losses are the accurate values of the given distribution system.

\section{PROPOSED METHODOLOGY}

The proposed approach that is based on Modified Cuckoo bird search method for initial sorting and Bird Swarm Optimisation for finding Optimum location and Size there by, modulating the final size and location with respect to the losses corresponding with the total cost of operation is as follows.

Step 1: Read line/branch data and Load/bus data of given radial distribution power system.
Step 2: Load flows are calculated by using Backward-Forward Sweep algorithm and total real power losses are calculated using simple real loss formulae mentioned in equation (3)

Step 3: Initiate Bird swarm or particles with given constraints and losses without DG as the maximum loss and consider the initial random position and initial random size as the best sizes.

Step 4: Now power flow calculation is done by ForwardBackward sweep algorithm and find system bus voltages, total real power losses are found.

Step 5: Objective function is evaluated and the results of ultimate optimum locations of different number of DG's and the sizes of the same are compared with the initial best values of location and size using equations (8) and (9) for minimum Objective function

Step 6: Compare the result of losses of each individual in the swarm such that the best position and size are the personal/present best position and personal/present best size among each individual's own position and size in $\mathrm{N}$ dimensional space.

Step 7: Now for sorting out the best pairs of values of position and size are taken by using Modified Cuckoo bird search method.

Step 8: Now compare all the results of best pairs of best positions and sizes are compared and the best solution is attained and the final position and the final size are called as the Global best location/position of number of DG's taken and Global best size of different number of DG's taken as per requirement.

Step 9: The Global best position and size at each iteration are evaluated for Objective function obeying the constraints.

Step 10: Now initiate the size modulator algorithm by giving the global best position and size and losses.

Step 11: Output of size modulator algorithm are the final accurate and the ultimate Optimum values of Position and sizes for DG installations.

\section{SIMULATION RESULTS}

The proposed methodology is applied on IEEE 33 standard system and IEEE 69 standard system using MATLAB software considered Base MVA $=100$, Base KV = 12.66. The proposed methodology is been applied/tested on two different test systems both at unity power factor i.e., Type 1 Generator which is able to deliver only the real power calculated for overall distribution power system. The results of the test bus systems are tabulated below.

\section{BSO PARAMETERS:}

Table 1: BSO Parameters

\begin{tabular}{|c|c|}
\hline Population per swarm & 50 \\
\hline Maximum Iterations & 10 \\
\hline Weighted coefficient & 1.36 \\
\hline $\mathrm{C} 1$ & 2 \\
\hline $\mathrm{C} 2$ & 2 \\
\hline
\end{tabular}


International Journal of Engineering and Advanced Technology (IJEAT) ISSN: 2249 - 8958, Volume-8, Issue-6S3, September 2019

\begin{tabular}{|c|c|}
\hline $\mathrm{r} 1, \mathrm{r} 2$ & Rand number $[0,1]$ \\
\hline Number of runs & $<5$ \\
\hline
\end{tabular}

TEST SYSTEM 1:

\section{IEEE 33 BUS SYSTEM:}

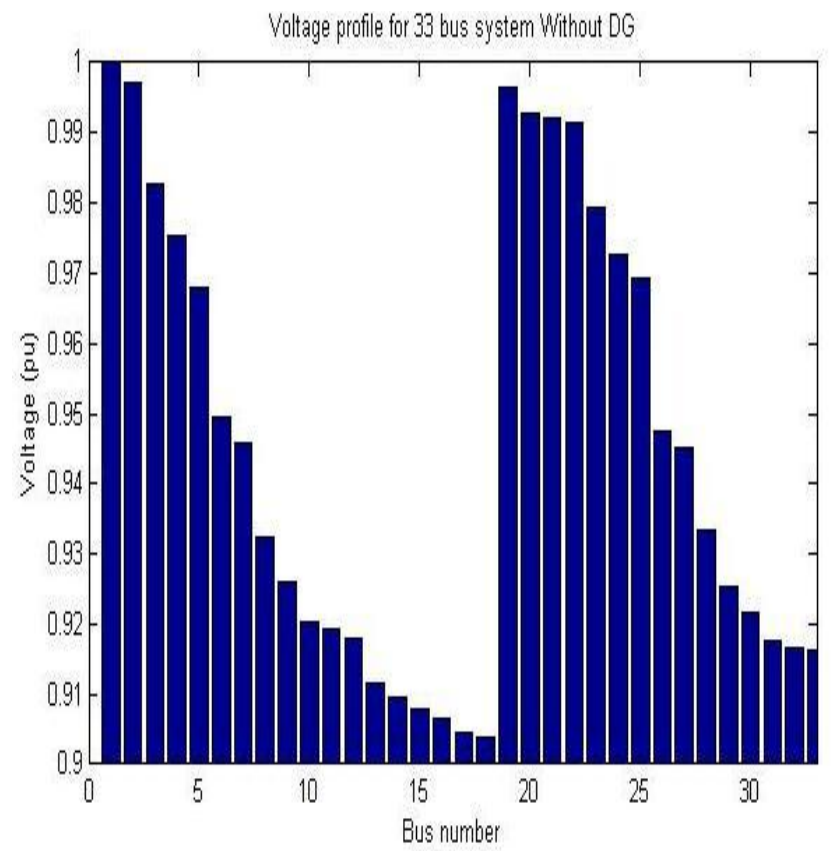

Figure 1 Base case Voltage trace of 33 bus system without DG

The 33 bus system is considered as the test bus system number 1 with a total active power load calculated adding all the real load data from test data is about $3.72 \mathrm{MW}$, and total reactive power load calculated by addition all reactive loads of each bus taken from bus/load data is about 2.3 MVAR which consists of 33 buses and 32 branches. The Voltage profile of 33 bus system when no DG is installed is shown in figure 1 in which the active losses without the installation DG is $210.83 \mathrm{KW}$ and the minimum voltage is found to be 0.9039 at bus 18 .

The active power losses are reduced to a greater extent thereby improving the voltage profile at each bus using proposed method are shown below. By means of calculating loss sensitivity factor analysis top 10 critical buses are selected for the placement of DG such that it reduces the time for the selection of buses for placement as done in previous optimisation methods. The loss sensitivity factors and 10 critical buses for 33 bus system are tabulated in Table 1. Out of all the 10 critical buses three buses are allocated as per the requirement of number of DG's with respect to their Optimal size by main CWFBSO.

Loss Sensitive Factors for 33 bus system:

Table 2: Loss factors of 33 bus system

\begin{tabular}{|c|c|c|}
\hline Bus number & Lsf & Vsf \\
\hline 6 & 0.0233 & 0.9994 \\
\hline 8 & 0.0215 & 0.9814 \\
\hline 28 & 0.0121 & 0.9827 \\
\hline
\end{tabular}

\section{VOLTAGE PROFLLE COMPARISON OF 33 BUS SYSTEM @ UNITY POWER FACTOR}

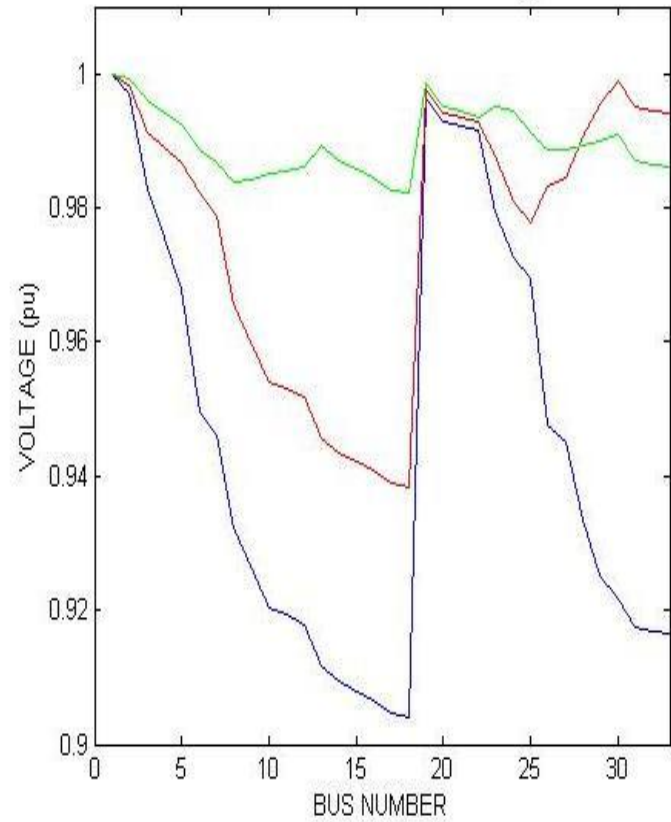

Figure 2: Voltage trace comparison of 33 bus system @ unity $\mathrm{PF}$

TEST SYSTEM 2:

\section{IEEE 69 BUS SYSTEM:}


Optimal Size \& location of Distributed Generation Using Bird Swarm Optimization with Cuckoo Search Sorting Algorithm

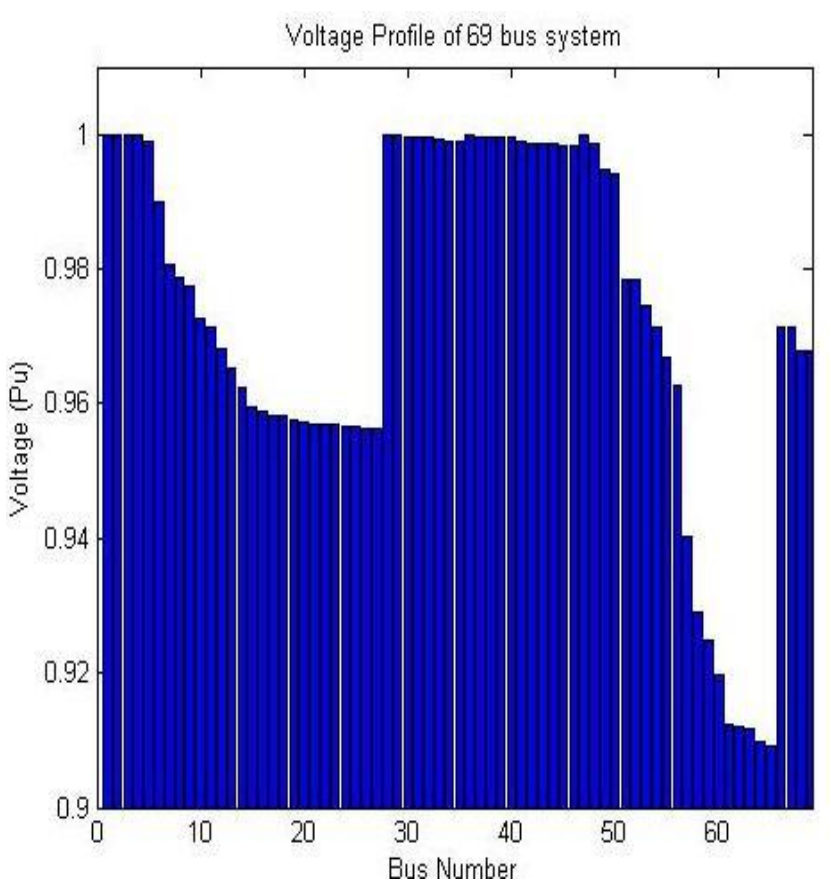

Figure 3: Base case Voltage trace of 69 bus system without DG

The 69 bus system is considered as the test bus system number 2 with a total active power load calculated adding all the real load data from test data is about $3.8 \mathrm{MW}$, and total reactive power load calculated by addition all reactive loads of each bus taken from bus/load data is about 2.69 MVAR which consists of total 69 buses in given system and total 68 branches in the system. The Voltage trace of 69 bus system when no DG is installed is shown in figure 3 in which the active power losses without DG is $224.98 \mathrm{KW}$ and the minimum voltage is found to be 0.9092 at bus number 65 .

The active power losses are reduced to a greater extent thereby improving the voltage profile at each bus using proposed method are shown below. By means of calculating loss sensitivity factor analysis top 10 critical buses are selected for the placement of DG such that it reduces the time for the selection of buses for placement as done in previous optimisation methods. The loss sensitivity factors and 10 critical buses for 69 bus system are tabulated in Table 3. Out of all the 10 critical buses three buses are allocated as per the requirement of number of DG's with respect to their Optimal size by main CWFBSO.

Loss Sensitive factors for 69 bus system:

Table 3: Loss Sensitive factors for 69 bus system

\begin{tabular}{|c|c|c|}
\hline Bus number & Lsf & Vsf \\
\hline 57 & 0.0373 & 0.9896 \\
\hline 58 & 0.0188 & 0.9779 \\
\hline 61 & 0.0119 & 0.9604 \\
\hline
\end{tabular}

Table 4 Results of 33 bus distribution system without installing DG and with DG @ Unity PF

\begin{tabular}{|l|l|l|l|c|}
\hline Number of DG's & Optimal Location & $\begin{array}{c}\text { Optimal Size of DG } \\
\text { (MW) }\end{array}$ & $\begin{array}{c}\text { Real Power Losses } \\
(\mathrm{KW})\end{array}$ & $\begin{array}{c}\text { Loss Reduction } \\
(\%)\end{array}$ \\
\hline Without DG & -- & -- & 210.83 & -- \\
\hline 1 DG & 6 & 2.31 & 111.1 & 47.3 \\
\hline
\end{tabular}




\begin{tabular}{|c|c|c|c|c|}
\hline 2 DG's & $\begin{array}{l}29 \\
10 \\
\end{array}$ & $\begin{array}{l}0.84 \\
0.89 \\
\end{array}$ & 92.33 & 56.2 \\
\hline 3 DG's & $\begin{array}{l}30 \\
13 \\
25\end{array}$ & $\begin{array}{l}0.97 \\
0.75 \\
0.89\end{array}$ & 74.2 & 64.8 \\
\hline
\end{tabular}

Table 5: Results of 69 bus distribution system without installing DG and with DG @ Unity PF

\begin{tabular}{|l|l|l|l|l|}
\hline Number of DG's & Location & $\begin{array}{l}\text { DG size } \\
(\mathrm{MW})\end{array}$ & $\begin{array}{l}\text { Real Power loss } \\
(\mathrm{KW})\end{array}$ & $\begin{array}{l}\text { Loss Reduction } \\
(\%)\end{array}$ \\
\hline Without DG & -- & -- & 224.98 & -- \\
\hline 1 DG & 61 & 1.74 & 83.8 & 62.7 \\
\hline 2 DG & 59 & 0.57 & 83.1 & 63.06 \\
& 61 & 1.29 & & \\
& 61 & 1.10 & 72.03 & 68 \\
\end{tabular}

\section{CONCLUSION}

With respect to the Crucial considerations of DG sizing and allocation, the convergence criteria and DG size allocation with economical operation is also should be priority in DG allocation. In this paper from obtained results tabulated in tables $4 \&$ table 5 an observation made that the proposed methodology has shown greater ability to reduce the losses as well for the improvement of voltage profile with respect to the economical DG size allocation using BSO with size modulator algorithm and greater saving of iteration run time is also observed by using the Modified Cuckoo bird algorithm. By the proposed method the voltage profile is improved greatly for 33 bus to a minimum voltage 0.9651 with a loss reduction percentage of 64.8 when three DG's are installed and for 69 bus distribution power system the voltage profile is greatly improved to the minimum voltage of 0.9875 with a loss reduction percentage of $68 \%$ which proves the superiority of the proposed methodology over the already existing methodologies both in the aspects of convergence and the DG size allocation.

\section{REFERENCES}

1. J. Federico, V. Gonzalez, and C. Lyra, "Learning classifiers shape reactive power to decrease losses in power distribution networks," in Proc. IEEE Power Eng. Soc. General Meet., Jun. 2005, vol. 1, pp. 557-562.

2. Georgilakis, P.S., Hatziargyriou, N.D.: 'Optimal distributed generation placement on power distribution networks: models, methods, and future research', IEEE Trans. Power Syst.,2013, 28, (3), pp. 3420-3428

3. Katsigiannis, Y.A., Georgilakis, P.S.: 'Effect of customer worth of interrupted supply on the optimal design of small isolated power systems with increased renewable energy penetration'. IET Gener. Transm. Disturb., 2013, 7, (3), pp.,265-275

4. Pecas Lopes, J.A., Hatziargyriou, N., mutale, J., Djapic, P.,Jenkis, N: 'Integrating distributed generation into electric power systems: a review of drivers, challenges and opportunities', Elect. Power Syst. Res., 2007, 77, (9), pp. 1189-1203

5. Jabr, R., Pal, B.: ' Ordinal optimisation approach for locating and sizing distributed generation', IET Gener, transm. Distrib., 2009, 3, (8). Pp. 713-723

6. R. K. Singh, and S. K. Gowsami, "Optimum allocation of distributed generations based on nodal pricing for profit, loss reduction, and voltage improvement including voltage rise issue", Elsevier, Science Direct, Electr. Power Energy Syst., 32, pp. 637-644, 2010.

7. M. N. Moradi, and M. Abedini, "A combination of genetic algorithm and particle swarm optimization for optimal DG location and sizing in distribution systems", Elsevier, Science Direct, Electr. Power Energy Syst., pp. 66-74, 2012.

8. S. K. Injeti, and N. P. Kumar, "A Novel Approach to Identify Optimal Access Point and Capacity of Multiple DGs in a Amall, Medium and Large Scale Radial Distribution Systems", Elsevier, Science Direct, Electr. Power Energy Syst., pp. 142-151, 2013.

9. Mohamed Imran A., and Kowsalya M., "Optimal size and siting of multiple distribution generators in distribution system using bacterial foraging optimization”, Elsevier, Science Direct, Swarm and Evolut. Comput., 15, pp. 58-65, 2014.

10. Mohammed $Z$ Amri Che Wanik and A.Mohamed,"Intelligent management of distributed generators for loss minimization and voltage control ",15th Intenational (MELCON) pp. 685-690, 2010.

11. A.Alsaadi , and B.Gholami, "An effective approach for distribution system power flow solution", Intern. Journ. of Electr. and Electron. Eng.. December, 2009

12. JEN-HAO TENG ''A Network-Topology-based Three-Phase Load Flow for Distribution Systems",, Vol. 24, No. 4, 2000. pp. 259-264

13. N.Acharya, P.Mahat, N.Mithulananthan,"An analytical approach for distributed generation allocation in distribution network ", Electr. Power and Energy Systems, pp. 669-678, 2006.

14. S. K. Injeti, V. K. Thunuguntla, and M. Shareef, "Optimal allocation of capacitor banks in radial distribution systems for minimization of real power loss and maximization of network savings using bio-inspired optimization algorithms", Elsevier Ltd, Electrical Power and Energy Systems 69, pp. 441-455, 2015.

15. J. Kennedy and R. C. Eberhart, "Particle swarm optimization", Proceedings of the IEEE International Conference on Neural Networks IV, Piscataway, NJ: IEEE Service Center, pp. 1942-1948, 1995.

16. Chintalapudi V.Suresh, M.S.Giridhar, "Analysing the effect of distributed generators on economical and technical aspects of distributed systems.

17. R. C. Eberhart and J. Kennedy, "A new optimizer using particles swarm theory", Proceedings of the 6th In-ternational Symposium on Micro Machine and Human Science, pp. 39-43, 4-6 October 1995. doi:10.1109/MHS.1995.494215.

18. Babu, T. Vandana, T. Satyanarayana Murthy, and B. Sivaiah. "Detecting unusual customer consumption profiles in power distribution systems-APSPDCL." 2013 IEEE International Conference on Computational Intelligence and Computing Research. IEEE, 2013.

19. P. V. Prasad, S. Sivanagaraju, and N. Sreenivasulu, "A fuzzy genetic algorithm for optimal capacitor placement in radial distribution systems", ARPN Journal of Engineering and Applied Sciences, Vol. 2, No. 3, 2007.

20. Pb Agus Ristono*,'Design Of Reliable And Efficient Manchester Carry Chain Adder Based 8-Bit Alu For High Speed Applications",Journal Of VLSI Circuits And Systems, 1 (01), 1-4,2019 